\title{
Smectic, nematic, and isotropic phases in binary mixtures of thin and thick hard spherocylinders
}

\author{
Giorgio Cinacchi ${ }^{\text {a) }}$ \\ Dipartimento di Chimica, Università di Pisa, Via Risorgimento 35, I-56126 Pisa, Italy \\ Yuri Martínez-Ratón \\ Grupo Interdisciplinar de Sistemas Complejos, Departamento de Matemáticas, Escuela Politécnica \\ Superior, Universidad Carlos III de Madrid, Avenida de la Universidad 30, E-28911 Leganés, \\ Madrid, Spain \\ Luis Mederos \\ Instituto de Ciencia de Materiales, Consejo Superior de Investigaciones Científicas, \\ E-28049 Cantoblanco, Madrid, Spain \\ Enrique Velasco \\ Departamento de Física Téorica de la Materia Condensada, Universidad Autónoma de Madrid, \\ E-28049 Cantoblanco, Madrid, Spain and Instituto de Ciencia de Materiales Nicolás Cabrera, \\ Universidad Autónoma de Madrid, E-28049 Cantoblanco, Madrid, Spain
}

\begin{abstract}
A second-virial Onsager theory, based on Parsons-Lee rescaling and suitably extended to deal with multicomponent systems and smectic phases, has been used to calculate the phase diagram of a collection of binary mixtures of thin and thick hard spherocylinders. In particular, two types of phase diagrams are investigated. First, a number of binary mixtures where the two components have the same total length have been considered; in addition, the phase diagram of a binary mixture where the two components have the same volume has been calculated. For the particles of one of the two components, the length of the cylindrical part and the diameter have always been set equal to 5 and 1 , respectively. Spherocylinders of the same total length and different diameter tend to demix considerably as soon as the diameter ratio deviates from unity. This happens especially at high pressures, when at least the phase richer in the thicker component is smectic. In the case where the two components have equal volumes, demixing is further increased due to the disparity not only in particle diameter but also in particle lengths. The incorporation of inhomogeneous layered phases is seen to alter significantly the phase diagrams calculated if only homogeneous phases are allowed, since transitions to a smectic phase often preempt those to a nematic or an isotropic phase. The apparent versatility of the recent experimental techniques suggests that the phase diagram features predicted by the theory might be also observed in real systems.
\end{abstract}

\section{INTRODUCTION}

Onsager theory ${ }^{1}$ is of fundamental importance for two reasons. ${ }^{2}$ First, it demonstrates that a phase transition can be driven by entropy alone; specifically, it explains the transition from a nematic $(N)$ to an isotropic $(I)$ phase, undergone by a system of very long and thin, freely rotating, hard rods, as a balance between ideal and packing entropies. Second, it is a density functional theory (DFT) ante litteram, which provides the exact free energy expression for this particular system. ${ }^{3}$

The idea that shape anisotropy is the sole ingredient necessary for the appearance of liquid-crystalline phases was confirmed by later theoretical calculations ${ }^{4,5}$ and computer simulations. ${ }^{6}$ These studies showed not only that a nematic phase can be thermodynamically stable even in systems of hard body particles of moderate aspect ratio $(\kappa)$, but also that

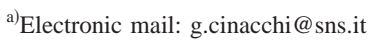

partially positionally ordered phases, such as smectic $(S)$ and columnar phases, can be generated in such systems.

The numerical results reviewed in Ref. 6 stimulated the search for a generalization of the Onsager theory able to account for them. In the case of pure hard body systems, for which unambiguously accurate phase boundaries have been traced out by computer simulation techniques, the approach due to Parsons ${ }^{7}$ and applied to hard spherocylinders (HSCs) by Lee, ${ }^{8}$ henceforth called PL theory, has emerged among a number of proposals as the most effective in providing sufficiently precise $I-N$ phase coexistence data for a variety of particle sizes and shapes, ${ }^{9-11}$ and also for systems with different dimensionalities. ${ }^{12}$ In addition, PL theory and a more elaborate extension to nonuniform phases proposed by Somoza and Tarazona ${ }^{13,14}$ are able to give a faithful representation of the thermodynamics of the $I-S$ and $N-S$ phase transitions in pure and binary HSC systems. ${ }^{15-18}$

Massive difficulties have precluded so far the accurate calculation by computer simulation techniques of the entire 
phase diagram of hard body particle mixtures. There is, anyway, an indication that PL theory might give reliable data also in the delicate case of a rod-plate binary mixture. ${ }^{19}$

Mixtures constitute the next natural extension of the Onsager theory; as already pointed out in Ref. 1, they are expected to exhibit an interesting phenomenology. The numerous theoretical studies performed on binary, ternary, and polydisperse mixtures, employing Onsager and PL theories, have confirmed Onsager's conjectures. In fact, strong fractionation, reentrance phenomena, and various demixing phase transitions, sometimes with associated critical points, have been observed. This body of literature has been partially reviewed in Ref. 20. In most of these studies only $I$ and $N$ phases are considered. However, a number of the aforementioned interesting phenomena are seen to occur at such high densities that more ordered phases should be also present. In a first attempt to tackle the complications accompanying the treatment of partially positionally ordered phases, an extension of PL theory to multicomponent layered phases has been formulated and applied to the calculation of the phase diagram of a few HSC mixtures with length bidispersity. ${ }^{17,18}$

This work is the continuation of those presented in Refs. 17 and 18, and deals with binary mixtures of thin and thick freely rotating HSCs. The motivation of this work is twofold. It is of interest to address the question of how permitting the existence of $S$ phases alters the phase diagram features observed when only homogeneous or perfectly orientationally ordered phases are allowed. ${ }^{21-31}$ In addition, this type of binary mixture is particularly interesting because its phase behavior might be mimicked by real experimental systems whose construction has been recently reported. ${ }^{30}$ The phase diagram topology of mixtures of particles whose interactions are of hard-body type is expected to crucially depend on geometric details of the molecules since only entropic considerations come into play in this case. Thus, the depletion effects that explain the observed phenomenology in mixtures of particles of the same diameter and different lengths can be significantly different when particles have different diameters but the same length. Therefore, a complete study of different types of mixtures is important in order to find relevant phenomenology that can be then explored from the experimental point of view.

PL theory is shortly reviewed in Sec. II. Its most obvious extension to inhomogeneous systems will be applied to the calculation of the phase diagram of HSC binary mixtures, whose components have either the same total length or the same volume. These results will be presented and discussed in Sec. III. The conclusions drawn from them are collected in Sec. IV, which also contains a few comments on possible future work.

\section{RELEVANT THEORETICAL EXPRESSIONS AND COMPUTATIONAL DETAILS}

In the extended PL theory, the configurational part of the free energy density, $f$, of a bidisperse mixture of particles forming, in the most general case, smectic $A$ and smectic $A_{2}$ phases $^{32}$ reads

$$
\begin{aligned}
\beta f= & \sum_{i=1}^{2} \frac{1}{\delta} \int_{0}^{\delta} d z \rho_{i}(z)\left[\ln \rho_{i}(z)-S_{i}^{\mathrm{rot}}\left(z ;\left[f_{i}\right]\right)-1\right] \\
& +\Psi(\phi) \sum_{i=1}^{2} \sum_{j=1}^{2} \frac{1}{\delta} \int_{0}^{\delta} d z \rho_{i}(z) \\
& \int_{-\infty}^{\infty} d \zeta \rho_{j}(z+\zeta) \alpha_{i j}\left(z, \zeta ;\left[f_{i}, f_{j}\right]\right),
\end{aligned}
$$

where the single-particle density distribution function of each component $\rho_{i}(z, \Omega)$ has already been decomposed, as usual, into a positional part $\rho_{i}(z)$ and an orientational function $f_{i}(z, \Omega)$. Here $z$ is the position of a particle along the director (this is the only spatial dependence expected given the symmetry of the phases to be studied), and the orientational variables are collected by the symbol $\Omega$. Also in the above expression, the first sum is the ideal contribution to the free energy density, while the following double sum is the approximate excess contribution to $f$. In Eq. (1) the variables $\beta$ and $\delta$ stand for the inverse thermal energy $\left(k_{B} T\right)^{-1}$ and smectic period, and the function $\Psi(\phi)$ is the CarnahanStarling factor, which is a function of the average packing fraction $\phi$. The orientational distribution function $f_{i}(z, \Omega)$ enters the definition of the orientational entropy, $S_{i}^{\text {rot }}$ and the quantity $\alpha_{i j}$, which is

$$
\begin{aligned}
\alpha_{i j}\left(z, \zeta ;\left[f_{i}, f_{j}\right]\right)= & \iint d \Omega d \Omega^{\prime} f_{i}(z, \Omega) f_{j}(z \\
& \left.+\zeta, \Omega^{\prime}\right) \int d \mathbf{R} M_{i j}\left(\mathbf{R}, \zeta, \Omega, \Omega^{\prime}\right),
\end{aligned}
$$

with $M_{i j}\left(\mathbf{R}, \zeta, \Omega, \Omega^{\prime}\right)$ being minus the Mayer function of particles $i$ and $j$ and $\mathbf{R}$ the set of coordinates perpendicular to $z$.

In principle, the minimization of Eq. (1) can be carried out by a number of different numerical methodologies. Among these, we mention the variational method based on parametrization of the single-particle densities, as in Ref. 1; direct minimization of the functional using some numerical minimization algorithm; numerical solution of the associated Euler-Lagrange equations, as in Ref. 33; and Monte Carlo simulated annealing techniques, as in Ref. 34. The first, although certainly not the most accurate, appears the only practicable route in the present case. It involves a judicious selection among the eligible parametrizations of the singleparticle densities. The choice made here is the same adopted previously in Refs. 17 and 18, where it is amply described. It transforms $f$ into a function of $\rho_{0}$, the total number density; $\lambda_{1}$ and $\lambda_{2}$, the two parameters which, together with $\delta$, enter the functional form adopted for $\rho_{1}(z)$ and $\rho_{2}(z)$, and which are related to the smectic order parameters $\tau_{1}$ and $\tau_{2}$, respectively; and $\eta_{1}$ and $\eta_{2}$, the two nematic order parameters. It rests upon two major approximations, which are, to a certain degree, intertwined.

First, positional and orientational variables are assumed to be always decoupled, i.e., $f_{i}(z, \Omega)=f_{i}(\Omega), \forall_{i}$. For a layered phase, this is not generally true, but it becomes a progressively good approximation as the particle shape becomes rodlike and uniaxial and the particle aspect ratio 
$\kappa_{i}=\left(L_{i}+D_{i}\right) / D_{i}$ (where $L_{i}$ is the particle's length and $D_{i}$ its diameter) is sufficiently far from unity. ${ }^{35,36}$ The meeting of these two conditions validates also the other major approximation made, namely,

$$
\alpha_{i j}\left(\zeta, \eta_{1}, \eta_{2}\right) \approx \alpha_{i j}\left(\zeta, \frac{\eta_{1}+\eta_{2}}{2}, \frac{\eta_{1}+\eta_{2}}{2}\right),
$$

which amounts to evaluating only the "diagonal" terms for all values of $\zeta$. The fulfillment of these conditions makes the population of particles located in the interlayer regions, which have a tendency to be aligned perpendicularly to the director, quite exiguous. Their contribution to the free energy density is therefore negligible.

In the case of mixtures, it is more convenient to use the Gibbs free energy density $g$ related to $f$ as follows:

$$
g=\frac{f+P}{\rho_{0}} .
$$

For any binary mixture investigated, this function is minimized with respect to the set of above-mentioned parameters for a large number of values of $x$, the mole fraction, and $P$, the pressure. For every value of $P$ considered, chemical potentials are then evaluated and properly equated to search for phase coexistence.

\section{RESULTS AND DISCUSSION}

Three quantities are needed to specify the state of a binary mixture of HSCs. Once we have set $D_{2}=1$, i.e., the diameter of the second component is taken as the unit of length, it remains to specify $L_{2}$, the length of the cylindrical part of the second component, and the two ratios $I=L_{1} / L_{2}$ and $d=D_{1} / D_{2}$. Every HSC binary mixture can be identified by the notation $\left(d, I, L_{2}\right)$. In all binary mixtures considered in the present work, the cylindrical length of the second, reference component has always been taken to have the paradigmatic value $L_{2}=5$.

In a first series of calculations, the first component possesses the same total length of the second, i.e., the binary mixtures investigated belong to the subclass $(d,(6-d) / 5,5)$. Several values of $d$ have been examined.

Figure 1 shows the phase diagram of the binary mixtures with $d=\frac{4}{3}$. The first component has $L_{1}=\frac{14}{3}$ and $D_{1}=\frac{4}{3}$, i.e., its aspect ratio is $\kappa=3.5$. Pure systems of HSCs with this aspect ratio undergo a transition from an isotropic phase directly into a smectic phase. The addition of HSCs with $L_{2}=5$ and $D_{2}=1$ slightly destabilizes the $S$ phase with respect to the $I$ phase. The most noteworthy feature of the phase diagram of the mixture $\left(\frac{4}{3}, \frac{14}{15}, 5\right)$ occurs at the other side of the mole fraction $x$ axis. The addition of the thicker component 1 to the thinner component 2 , which in pure form exhibits the sequence of phases $I-N-S$, destabilizes the $N$ phase with respect to the $I$ and $S$ phases. This fact is analogous to what is frequently observed in hard spherocylinder-hard spheres mixtures. ${ }^{37-39}$ The $I-N$ and $N-S$ phase boundaries then meet at a $S$ - $I-N$ triple point located at $P^{*}=\beta P D_{2}^{3} \simeq 1.25$ and $x_{S}$ $\simeq x_{N} \simeq x_{I} \simeq 0.2$.

The region of stability of a $S$ phase by adding component 1 to component 2 gradually diminishes as $D_{1}$ decreases

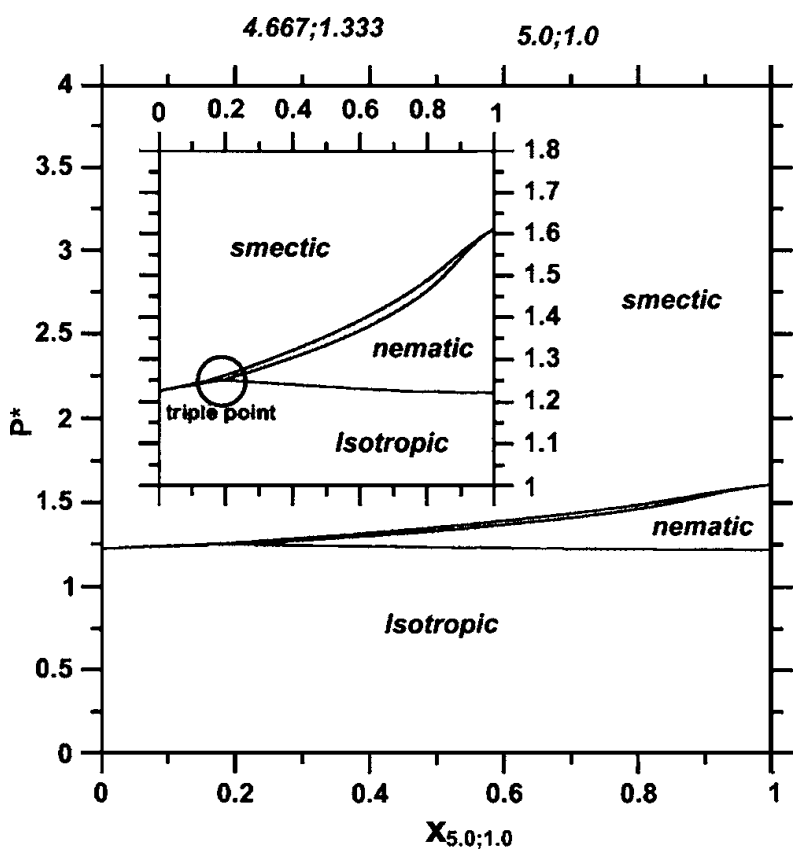

FIG. 1. Phase diagram of the binary mixture with $L_{1}=\frac{14}{3}, D_{1}=\frac{4}{3}, L_{2}=5$, and $D_{2}=1$. The inset shows details on the smectic-isotropic-nematic triple point.

while keeping $L_{1}+D_{1}=6$, until it naturally vanishes when the ideal binary mixture $(1,1,5)$ is reached. Further thinning of the diameter of component 1 leads to the opposite effect, as exemplified in Fig. 2(a), where the phase diagram of the binary mixture $(0.75,1.05,5)$ is plotted. Parts (b) and (c) of the same figure show the phase diagram of the mixtures $(0.5$, $1.1,5)$ and $(0.4,1.12,5)$, respectively. As $d$ diminishes, the $I-N$ phase boundaries naturally move toward higher pressures at the left side of the phase diagram, corresponding to compositions for which the thinner rods are more abundant; at the same time, the $I-N$ coexistence region gently enlarges. Similar effects pertain also to the $N-S$ phase transition but, in this case, the effect is quite rapid, with $P_{N-S}^{*}$ for the pure component 1 being larger than 4 for $d \leqslant 0.5$, while the $N-S$ coexistence region broadens considerably. When the aspect ratio $\kappa_{1}$ of the first component is larger than 3.5 , the $I-S$ transition disappears and is replaced by the $I-N$ transition and, as a result, the $I-N-S$ triple point also disappears. Further increase of $\kappa_{1}$ results in a broader region of $N$ stability at low concentration [Figs. 2(a) and 2(b)] but, for smaller values of $D_{1}, N-S$ demixing has the opposite effect of decreasing $N$ stability.

Further decreases of $d$ accentuate the phase diagram characteristics described above, as it can be appreciated in Figs. 3 and 4, where the phase diagram of the bidisperse mixtures $(0.3,1.14,5)$ and $(0.25,1.15,5)$, respectively, are plotted. Apart from the reentrant behavior of the $I-N$ phase transition appearing at $d=0.25$, a rather common phenomenon if the two components have sufficiently different sizes, there are two additional features worth to be noticed, namely, the $I-I$ and $N-N$ demixing lines. They are nonetheless metastable: for these values of $d, I-I$ demixing is already unstable with respect to nematic ordering, while $\mathrm{N}-\mathrm{N}$ demixing is preempted by the transition to the smectic phase. 

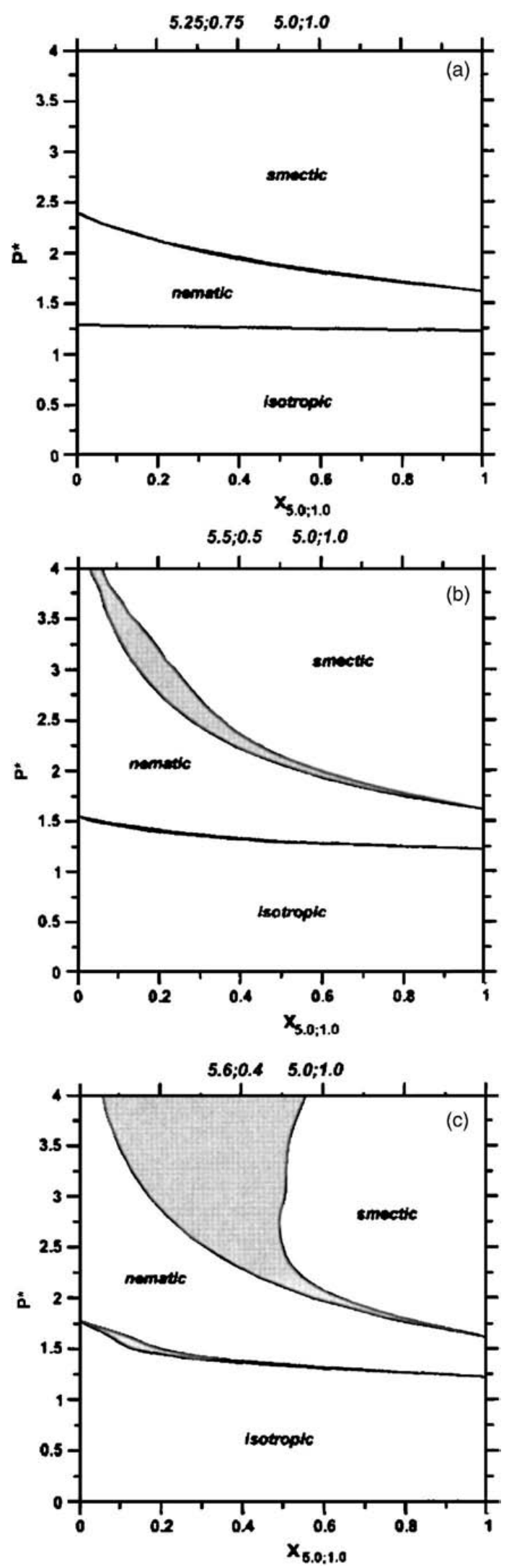

FIG. 2. (a) Phase diagram of the binary mixture with $L_{1}=5.25, D_{1}=0.75$, $L_{2}=5$, and $D_{2}=1$. (b) Phase diagram of the binary mixture with $L_{1}=5.5$, $D_{1}=0.5, L_{2}=5$, and $D_{2}=1$. (c) Phase diagram of the binary mixture with $L_{1}=5.6, D_{1}=0.4, L_{2}=5$, and $D_{2}=1$.
$5.7 ; 0.3 \quad 5.0 ; 1.0$

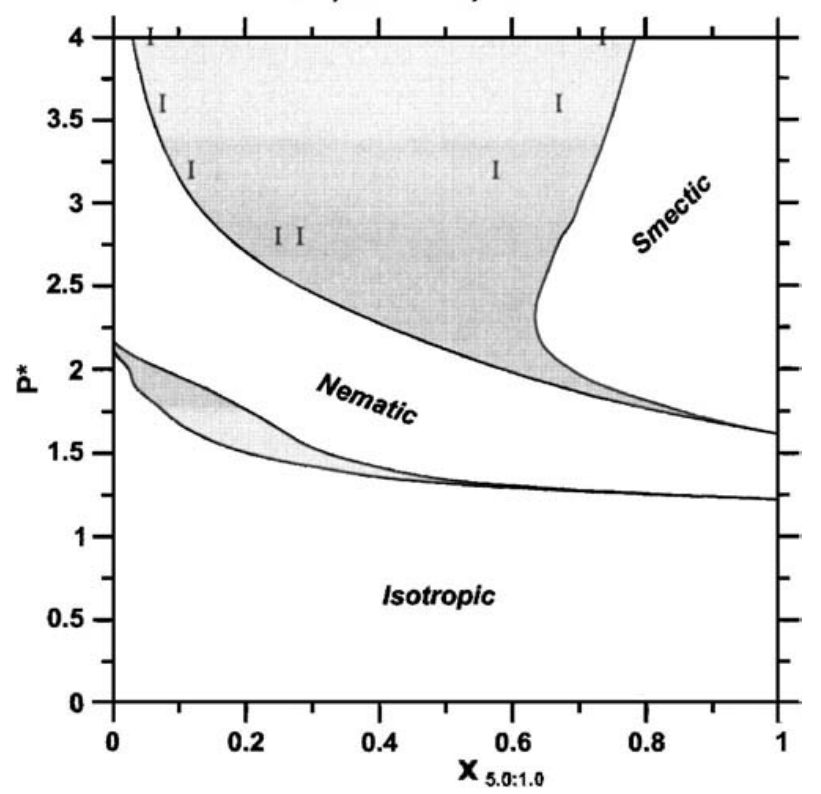

FIG. 3. Phase diagram of the binary mixture with $L_{1}=5.7, D_{1}=0.3, L_{2}=5$, and $D_{2}=1$. The letters $I$ indicate the metastable isotropic-isotropic demixing line.

The inclusion of smectic phases naturally modifies the phase diagram of HSC mixtures to a significant extent. One of the most representative examples is provided by the binary system $(0.2,1.16,5)$, whose liquid-crystalline phase behavior is shown in Fig. 5. The $N-N$ coexistence region, which would have existed at higher pressures, is replaced by a wider $N-S$ coexistence region; in place of an $I-N-N$ triple point, an $I-N-S$ triple point emerges at $P_{t 1}^{*} \simeq 2.9$ and $x_{I} \simeq 0$,

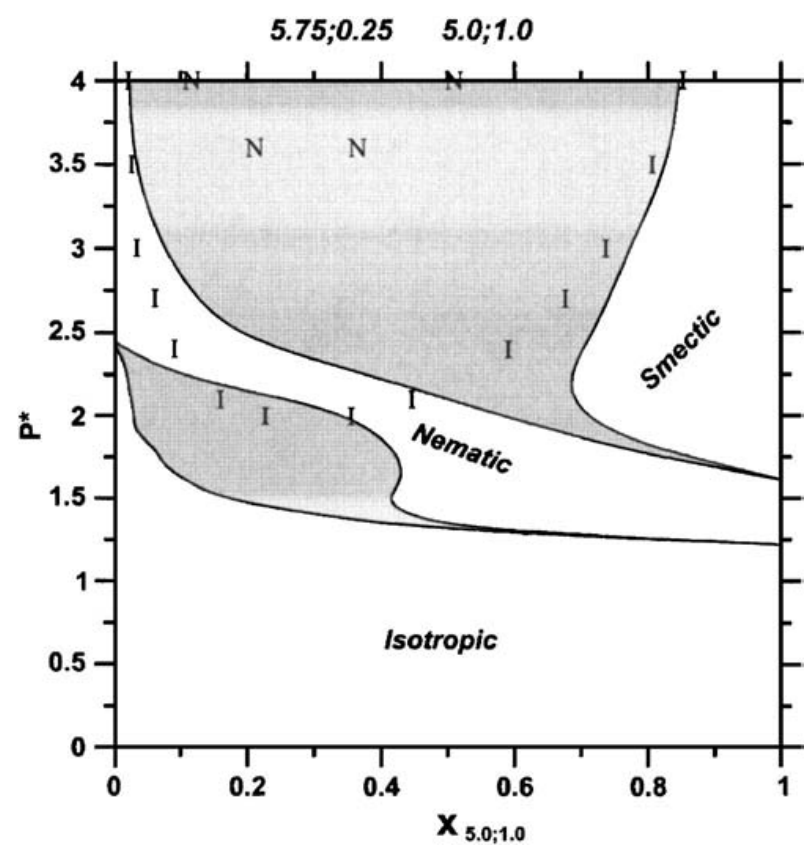

FIG. 4. Phase diagram of the binary mixture with $L_{1}=5.75, D_{1}=0.25, L_{2}$ $=5$, and $D_{2}=1$. The letters $I$ and $N$ indicate, respectively, the metastable isotropic-isotropic and nematic-nematic demixing lines. 


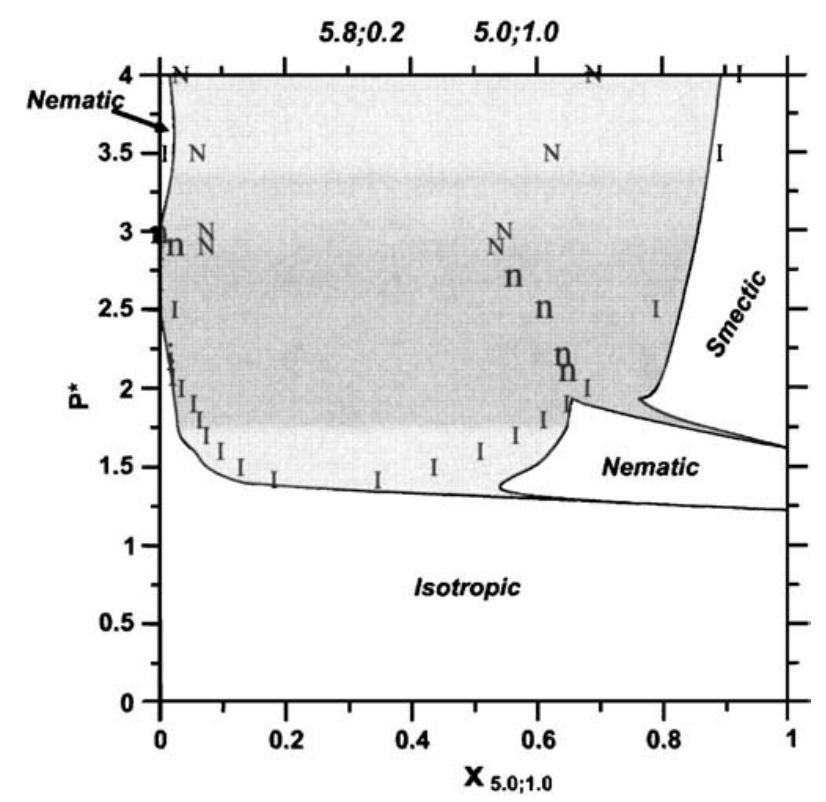

FIG. 5. Phase diagram of the binary mixture with $L_{1}=5.8, D_{1}=0.2, L_{2}=5$, and $D_{2}=1$. The letters $I$ indicate the metastable isotropic-isotropic demixing line; the letters $i$ and $n$ indicate the metastable isotropic-nematic boundary line; the letters $N$ indicate the metastable nematic-nematic demixing line.

$x_{N} \sim 0.0$, and $x_{S} \simeq 0.85$. As the pressure is further reduced, an isotropic phase, formed essentially by thin HSC particles only, coexists with a smectic phase of composition $x \simeq 0.8$, instead of coexisting with a nematic phase of composition $x \simeq 0.6$. At $P_{t 2}^{*} \simeq 1.9$, a second $I-N-S$ triple point occurs with compositions $x_{I} \simeq 0.02, x_{N} \simeq 0.65$, and $x_{S} \simeq 0.75$. At pressures lower than $P_{t 2}^{*}$, the $I-N$ coexistence region is unaltered when layered phases are taken into account, since the $N-S$ phase transition takes place at larger values of $x$. It is of interest to note that the metastable $I-I$ demixing line is almost in contact with the line delimiting the $I-N$ coexistence region at the isotropic side. It is therefore expected that a further decrease in $d$ would make a stable $I-I$ coexistence region to appear.

This is in fact the case, as can be observed in Figs. 6(a) and $6(\mathrm{~b})$, where the phase diagram of the binary mixtures $(0.15,1.17,5)$ and $(0.1,1.18,5)$, respectively, are plotted. The composition at which the $I-I$ critical point is located is roughly the same for the two systems, $x_{c} \simeq 0.2$. The pressure instead decreases monotonically with $d$. The $I-I$ coexistence region ends in an $I-I-N$ triple point. By further raising the pressure an isotropic phase, in which the thin HSCs are by far the majority component, is in equilibrium with a nematic phase composed prevalently by thick HSCs. The $I-N$ coexistence region is bounded above by an $I-N-S$ triple point. At pressures higher than this triple point, a quasipure isotropic phase of component 1 undergoes a transition to a smectic phase formed predominantly by component 2 . The phase diagrams of Fig. 6 are equivalent to each other, with the exceptions that the coexistence regions are wider for the system $(0.1,1.18,5)$, and that a reduced pressure of ca. 3.6 is enough for a HSC sample with $D_{1}=0.15$ and $L_{1}=5.85$ to
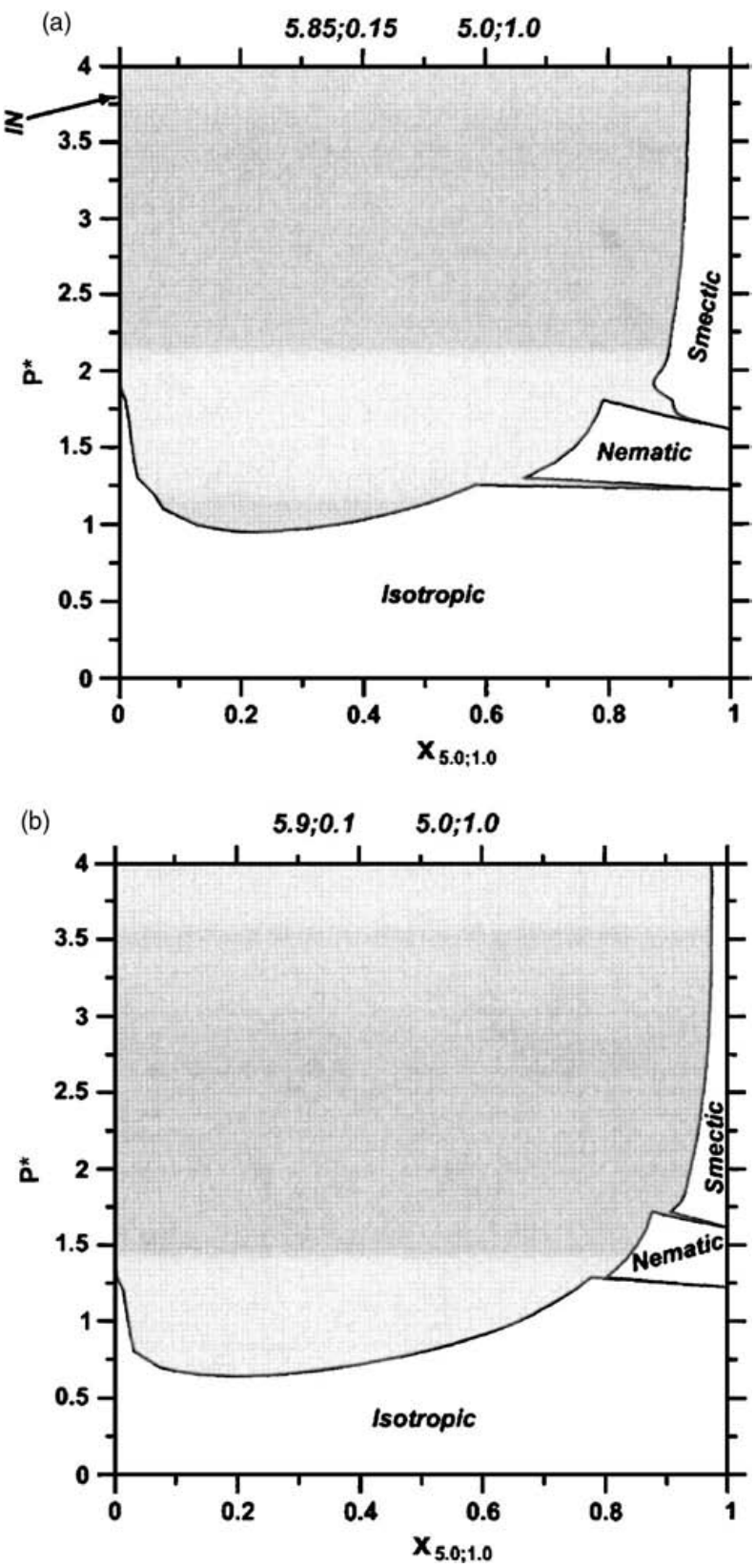

FIG. 6. (a) Phase diagram of the binary mixture with $L_{1}=5.85, D_{1}=0.15$, $L_{2}=5$, and $D_{2}=1$. (b) Phase diagram of the binary mixture with $L_{1}=5.9$, $D_{1}=0.1, L_{2}=5$, and $D_{2}=1$.

undergo a transition from an isotropic to a nematic phase, whereas at $P^{*}=4$ a HSC sample with $L_{1}=5.9$ and $D_{1}=0.1$ is still in an isotropic phase.

In the phase diagrams presented so far, the range of pressures shown is $[0,4]$ in reduced units. This is motivated by the fact that it is known from Monte Carlo computer simulations $^{40}$ that the reference component with $D_{2}=1$ and $L_{2}=5$ possesses a smectic-crystalline phase transition at $P^{*}$ $=2.343$. It is therefore expected that at higher pressures crystalline phases come into the picture, at least for the highest value of $x$. Neglecting them should lead to spurious phase diagrams. It is nevertheless of interest to explore the highest pressure part of the phase diagram, where the thinner com- 


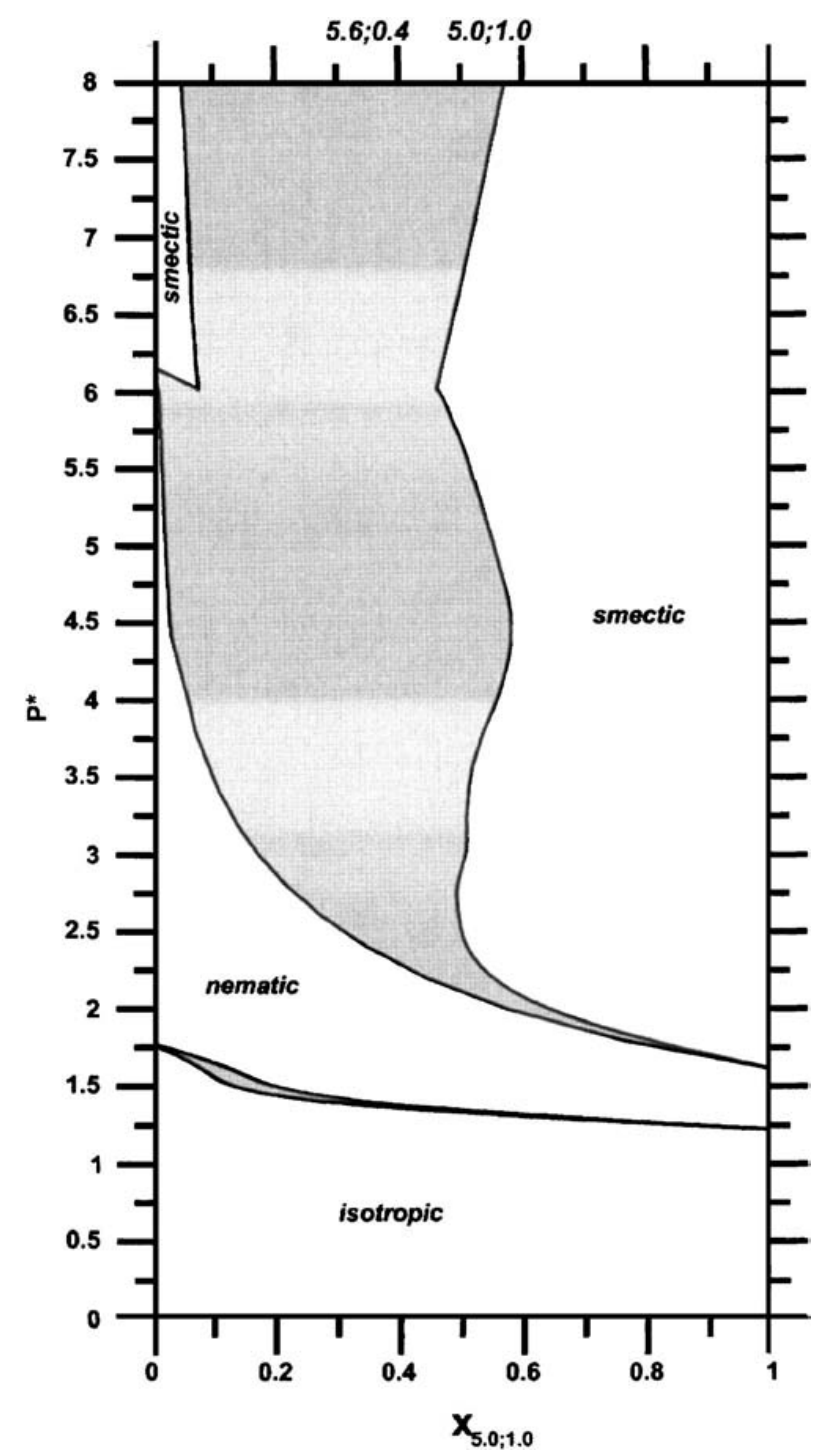

FIG. 7. Phase diagram of the binary mixture with $L_{1}=5.6, D_{1}=0.4, L_{2}=5$, and $D_{2}=1$ in the reduced pressure interval $[0 ; 8]$.

ponent 1 approaches and enters a smectic region. The binary system $(0.4,1.12,5)$ has been chosen to illustrate the evolution of a phase diagram at very high pressures. The corresponding phase diagram with $P^{*}$ in the interval $[0,8]$ is shown in Fig. 7. The competition among $N$-S coexistence, $S$ ordering, and $S$ - $S$ demixing resembles that among $I-N$ coexistence, $N$ ordering, and $N-N$ demixing observed for mixtures bidisperse in diameter, with diameter ratio sufficiently far from unity. A reentrant behavior is seen across $P^{*} \simeq 4.5$, while a $N-S-S$ triple point sets in at $P^{*} \simeq 6$, with compositions $x_{N} \simeq 0, x_{S} \simeq 0.07$, and $x_{S} \simeq 0.43$. The value of the triplepoint pressure is slightly below that at which a pure system of HSCs with $D_{1}=0.4$ and $L_{1}=5.6$ undergoes a $N-S$ phase transition. This means that in the vicinity of this phase transition, the addition of thicker, equally long HSCs induces a $S$ phase from a $N$ phase of thin HSCs. The width of the $S$ - $S$ coexistence region is smaller than that between $N$ and $S$

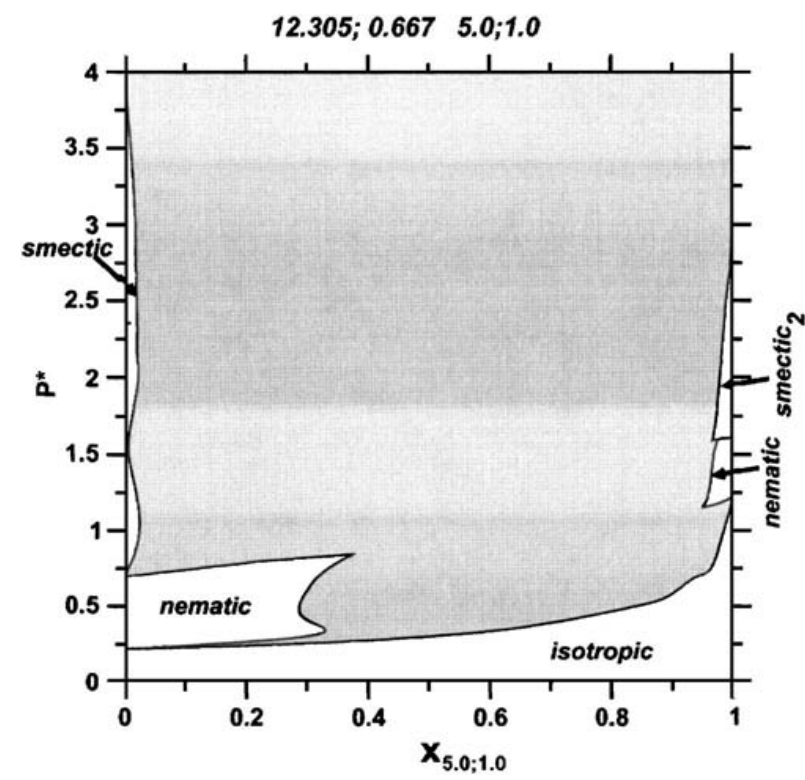

FIG. 8. Phase diagram of the binary mixture with $L_{1}=\frac{443}{180}, D_{1}=\frac{2}{3}, L_{2}=5$, and $D_{2}=1$.

phases, presumably because of the reduction in the difference between positional entropies of the two coexisting phases.

The last binary mixture examined is formed by a HSC with $L_{1}=\frac{443}{36}$ and $D_{1}=\frac{2}{3}$, and a HSC of $L_{2}=5$ and $D_{2}=1$, identified by the notation $\left(\frac{2}{3}, \frac{443}{180}, 5\right)$. The two constituent particles have the same volume. Figure 8 shows its phase diagram. Diameter and length bidispersity play together to increase the demixing tendency. In accordance with previous studies, ${ }^{17,18}$ the layered phase at larger value of $x$ is $S_{2}$ with the longer and thinner solute particles arranged parallel to the director in the interstices between layers. Curiously, the $S$ coexistence line at the other extreme of mole fractions is moderately undulating. This is probably caused by the fact that the $S$ phase, in which the longer and thinner rods are the majority components, is alternately in equilibrium with a sequence of phases of different symmetry: nematic, isotropic, nematic again, and smectic $A_{2}$, on raising pressure and passing through two $S-N-I$ and one $S-N-S_{2}$ triple points.

As can be seen from the figure, the reentrant behavior of the $I-N$ coexistence is inverted with respect to the other phase diagrams. This can be explained as follows: (i) the aspect ratio $\kappa_{1}$ is large enough as to lead to a broad region of $N$ stability between $I$ and $S$ phases, and (ii) as the volumes of the particles of the two components become similar, the pressure at which the smectic phase of the first component becomes stable is lower than in the other cases. This gives a more symmetrical phase diagram, as can be noticed from the figure.

\section{CONCLUSIONS}

In this work, the phase diagram of a number of binary mixtures of thin and thick hard spherocylinders has been computed, making use of an extension of the Parsons-Lee theory and including in the calculations smectic phases be- 
sides the nematic and isotropic phases. Within the entire space spanned by hard spherocylinder binary mixtures, two particular types have been selected for investigation. The first, a few cases of which have been examined, encompasses systems composed of two types of particles having the same total length and different diameters. The second, to which a typical example has been given attention, involves two types of particles having the same volume. In all cases, one of the component has been chosen to have the length of the cylindrical part equal to 5 and the diameter equal to 1 . The other component is thus univocally determined by the diameter ratio. The inclusion of smectic phases naturally modifies, to a significant extent, the topology of the phase diagram of all hard spherocylinder binary mixtures previously calculated without taking into account positionally inhomogeneous phases. Smectic phases do appear at sufficiently high pressures. In the first series of calculations, they are always of the $A$ type. As the diameter ratio deviates from unity, an equilibrium between a nematic phase very rich in thin rods and a smectic phase rich in thick rods is established at high yet realistic pressures, preempting nematic-nematic demixing. In the case of different constituent particles with the same volume, length and diameter disparities act synergically to expand the coexistence region between a smectic $A$ phase, very rich in thinner and longer rods, and a smectic $A_{2}$ phase, very rich in thicker and shorter rods, in which the minority component arranges parallel to the director in the interstices between layers.

Recent experimental techniques seem to have opened the way for the realization of rodlike particle mixtures of welldefined bidispersity. ${ }^{30}$ It would be very interesting to construct real binary mixtures whose components have geometrical parameters comparable to those examined in the present work, and then confront their phase behavior with those predicted by the present theory. Conversely, experiments suggest that other binary mixtures deserve to be studied theoretically, particularly those having a longer reference component. Comparisons between experiments and calculations employing the hard spherocylinder model imply that the latter is admitted to be realistic. However, it is not yet clear if this is the case. Investigations employing the hard spherocylinder model enriched with additional features, such as nonadditivity, ${ }^{41}$ and, most notably, employing flexible particles, are therefore very desirable. Finally, it would be nice to confront results from the extended Parsons-Lee theory for binary mixtures with those obtained using a fundamentalmeasure-based theory for freely rotating hard spherocylinders. Work along this line has been limited so far to either homogeneous, monodisperse, freely rotating particle systems, ${ }^{11}$ or to isotropic, ${ }^{42}$ perfectly ordered, ${ }^{42}$ Zwanzig $^{43}$ binary mixtures.

\section{ACKNOWLEDGMENTS}

The authors thank MIUR (Italy) and Ministerio de Educación y Ciencia (Spain) for financial support under the 2005 binational integrated program. They also gratefully acknowledge financial support from Ministerio de Educación y Cien- cia under Grant Nos. FIS2005-05243-C02-01, FIS200505243-C02-02, FIS2004-05035-C03-02, and BFM20030180, and from Comunidad Autónoma de Madrid (S-0505/ ESP-0299). One of the authors (Y.M.R.) was supported by a Ramón y Cajal research contract from the Ministerio de Educación y Ciencia.

${ }^{1}$ L. Onsager, Ann. N.Y. Acad. Sci. 51, 627 (1949).

${ }^{2}$ D. Frenkel, Theor. Chem. Acc. 103, 212 (2000).

${ }^{3}$ E. Velasco and P. Padilla, Mol. Phys. 94, 335 (1998).

${ }^{4}$ M. Hosino, H. Nakano, and H. Kimura, J. Phys. Soc. Jpn. 46, 1709 (1979).

${ }^{5}$ M. Hosino, H. Nakano, and H. Kimura, J. Phys. Soc. Jpn. 47, 740 (1979).

${ }^{6}$ M. P. Allen, G. T. Evans, D. Frenkel, and B. M. Mulder, Adv. Chem. Phys. 86, 1 (1993).

${ }^{7}$ J. D. Parsons, Phys. Rev. A 45, 5605 (1979)

${ }^{8}$ S. D. Lee, J. Chem. Phys. 87, 4972 (1987).

${ }^{9}$ S. Varga and I. Szalai, Mol. Phys. 98, 693 (2000).

${ }^{10}$ E. de Miguel and E. del Rio, J. Chem. Phys. 115, 9072 (2001).

${ }^{11}$ G. Cinacchi and F. Schmid, J. Phys.: Condens. Matter 14, 12223 (2002), and references therein.

${ }^{12}$ S. Varga and I. Szalai, J. Mol. Liq. 85, 11 (2000).

${ }^{13}$ A. M. Somoza and P. Tarazona, Phys. Rev. Lett. 61, 2566 (1988).

${ }^{14}$ A. M. Somoza and P. Tarazona, Phys. Rev. A 41, 965 (1990).

${ }^{15}$ M. Esposito and G. T. Evans, Mol. Phys. 83, 835 (1994).

${ }^{16}$ E. Velasco, L. Mederos, and D. E. Sullivan, Phys. Rev. E 62, 3708 (2000).

${ }^{17}$ G. Cinacchi, E. Velasco, and L. Mederos, J. Phys.: Condens. Matter 16, S2003 (2004).

${ }^{18}$ G. Cinacchi, L. Mederos, and E. Velasco, J. Chem. Phys. 121, 3854 (2004).

${ }^{19}$ P. J. Camp and M. P. Allen, Physica A 229, 410 (1996).

${ }^{20}$ H. H. Wensink and G. J. Vroege, J. Phys.: Condens. Matter 16, S2015 (2004).

${ }^{21}$ R. P. Sear and G. Jackson, J. Chem. Phys. 103, 8684 (1995).

${ }^{22}$ R. P. Sear and B. M. Mulder, J. Chem. Phys. 105, 7727 (1996).

${ }^{23}$ R. van Roij and B. Mulder, Phys. Rev. E 54, 6430 (1996).

${ }^{24}$ M. Dijkstra and R. van Roij, Phys. Rev. E 56, 5594 (1997).

${ }^{25}$ R. van Roij, B. Mulder, and M. Dijkstra, Physica A 261, 374 (1998).

${ }^{26}$ P. C. Hemmer, Mol. Phys. 96, 1153 (1999).

${ }^{27}$ P. C. Hemmer, J. Stat. Phys. 100, 3 (2000).

${ }^{28}$ P. C. Hemmer and T. H. Marthinsen, Mol. Phys. 100, 667 (2002).

${ }^{29}$ S. Varga, A. Galindo, and G. Jackson, Mol. Phys. 101, 817 (2003).

${ }^{30}$ K. R. Purdy, S. Varga, A. Galindo, G. Jackson, and S. Fraden, Phys. Rev. Lett. 94, 057801 (2005).

${ }^{31}$ S. Varga, K. Purdy, A. Galindo, S. Fraden, and G. Jackson, Phys. Rev. E 72, 051704 (2005).

${ }^{32}$ No other positionally inhomogeneous phases, such as columnar and crystalline phases, have been contemplated in the present work. The extended PL theory is not expected to be not able to deal with crystalline phases, for which a more elaborate theory is to be used. Even if PL theory could be able to describe columnar phases, the presence of the latter as thermodynamically stable phases in a binary mixture of thick and thin hard rods is unlikely.

${ }^{33}$ J. Herzfeld, A. E. Berger, and J. Wingate, Macromolecules 17, 1718 (1984).

${ }^{34}$ D. C. Williamson and G. Jackson, Mol. Phys. 83, 603 (1994).

${ }^{35}$ R. van Roij, P. Bolhuis, B. Mulder, and D. Frenkel, Phys. Rev. E 52, R1277 (1995)

${ }^{36}$ G. Cinacchi, Chem. Phys. Lett. 416, 238 (2005).

${ }^{37}$ T. Koda, M. Numajiri, and S. Ikeda, J. Phys. Soc. Jpn. 65, 3551 (1996).

${ }^{38}$ Z. Dogic, D. Frenkel, and S. Fraden, Phys. Rev. E 62, 3925 (2000).

${ }^{39}$ F. J. Vesely, Mol. Phys. 103, 679 (2005).

${ }^{40}$ P. Bolhuis and D. Frenkel, J. Chem. Phys. 106, 666 (1997).

${ }^{41}$ K. Shundyak, R. van Roij, and P. van der Schoot, J. Chem. Phys. 122, 094912 (2005).

${ }^{42}$ A. Perera, J. Mol. Liq. 109, 73 (2004).

${ }^{43}$ Y. Martínez-Ratón, E. Velasco, and L. Mederos, J. Chem. Phys. 123, 104906 (2005). 